\title{
SMALL VALUES OF COEFFICIENTS OF A HALF LERCH SUM
}

\author{
XINHUA XIONG
}

\begin{abstract}
Andrews, Dyson and Hickerson proved many interesting properties of coefficients for a Ramanujan's $q$-hypergeometric series by relating it to real quadratic field $\mathbb{Q}(\sqrt{6})$ and using the arithmetic of $\mathbb{Q}(\sqrt{6})$, hence solved a conjecture of Andrews on the distributions of its Fourier coefficients. Motivated by Andrews's conjecture, we discuss an interesting $q$-hypergeometric series which comes from a Lerch sum and rank and crank moments for partitions and overpartitions. We give Andrews-like conjectures for its coefficients. We obtain partial results on the distributions of small values of its coefficients toward these conjectures.
\end{abstract}

\section{INTRODUCTION AND STATEMENT OF RESULTS}

The function

$$
\begin{aligned}
\sigma(q) & =\sum_{n=0}^{\infty} S(n) q^{n}=1+\sum_{n=1}^{\infty} \frac{q^{\frac{n(n+1)}{2}}}{(-q)_{n}} \\
& =1+q-q^{2}+2 q^{3}+\cdots+4 q^{45}+\cdots+6 q^{1609}+\ldots
\end{aligned}
$$

first appeared in Ramanujan's lost note book [20]. It was noted that $S(n)$ can be interpreted as the number of partitions of $n$ into distinct parts with even rank minus the number with odd rank. Recall that Dyson's rank of a partition is defined as its largest part minus its number of parts. Although it has a partition interpretation, it behaves completely different from most of partition functions. Andrews [2] noted that most $q$-series with partition interpretations either have coefficients which tend to infinity in absolute value or are bounded. For instance, the usual partition function $p(n)$, whose generating function can be represented as a hypergeometric series, grows very fast.

In his paper [2], Georg Andrews studied $S(n)$ and conjectured that

(1) $S(n)$ is zero infinitely often,

(2) $\limsup |S(n)|=+\infty$.

Andrews's conjecture was soon proved by himself, Dyson and Hickerson [5]. They related $\sigma(q)$ to the arithmetic of quadratic field $\mathbb{Q}(\sqrt{6})$. They obtained an exact formula for $S(n)$, which implies that $S(n)$ is lacunary, i.e. its coefficients are almost always zero, and attains every positive integers infinitely many times.

2000 Mathematics Subject Classification. 11P82, 11N37.

Key words and phrases. $q$-hypergeometric series, coefficients of $q$-series, lacunary, bound.

The author was supported by the Austria Science Foundation (FWF) grant SFB F50-06 (Special Research Programm "Algorithmic and Enumerative Combinatorics"). 
Besides the strange behaviors of its coefficients, the function $\sigma(q)$ is found related to automorphic forms. Cohen [10] used it to construct a classical Maass wave forms. It has a representation as Hecke-type double sums

$$
\sigma(q)=\sum_{\substack{n \geq 0 \\|j| \leq n}}(-1)^{n+j} q^{\frac{n(3 n+1)}{2}-j^{2}}\left(1-q^{2 n+1}\right) .
$$

Zwegers [22] used Hecke-type double sums to describe mock theta functions, so Bringmann and Kane [7] viewed it as a "false mock theta function". Along this line, more examples resembling $\sigma(q)$ are found and relations to quadratic fields and automorphic forms are built, see [8, [9], [11, [14, [16] and [17] etc..

In this paper, we will not go to the directions to relate more $q$-hypergeometric series to quadratic fields or automorphic forms. We will turn to the properties (1) and (2) considered by Andrews but for another strikingly simple-looking $q$-hypergeometric series which is defined by

$$
h(q):=\sum_{n=1}^{\infty} h(n)=\sum_{n=1}^{\infty} \frac{(-1)^{n+1} q^{n(n+1) / 2}}{1-q^{n}} .
$$

$h(q)$ looks like $\sigma(q)$, and the distributions of its coefficients $h(n)$ appears to be similar to that of $S(n)$ in some faces. But in other faces, it completely does not. We will prove that $h(q)$ has property (1), but it is not lacunary. We conjecture it has property (2). We note that $h(q)$ is related to mock theta functions. Because the completed sum

$$
\sum_{n=-\infty}^{-1} \frac{(-1)^{n+1} q^{n(n+1) / 2}}{1-q^{n}}+\sum_{n=1}^{\infty} \frac{(-1)^{n+1} q^{n(n+1) / 2}}{1-q^{n}}
$$

is almost the specialization of Lerch sum

$$
\sum_{n \in \mathbb{Z}} \frac{(-1)^{n} e^{\pi i\left(n^{2}+n\right) \tau+2 \pi i n v}}{1-e^{2 \pi i n \tau+2 \pi i u}}
$$

by taking $u=v=0$ and letting $q=e^{2 \pi i \tau}$. This is why we call $h(q)$ a half Lerch sum. Lerch sums are building blocks for mock theta functions by the works of Zwegers [22]. Secondly, $h(q)$ has a similar Hecke-type double sums form

$$
h(q)=\sum_{\substack{n \geq 1 \\ 1 \leq j \leq n-1}} q^{n^{2}+n j}+\sum_{\substack{n \geq 1 \\ 0 \leq j \leq n}} q^{n^{2}+n j} .
$$

But this double sum is different from usual Hecke double sums, here $j$ appears linearly in the power of $q$. Moreover, $h(q)$ is related to the first positive crank moments for partitions and overprtitions. By [4], the generating function of the first positive crank moments for partitions can be represented as

$$
\frac{1}{(q)_{\infty}} \sum_{n=1}^{\infty} \frac{(-1)^{n+1} q^{n(n+1) / 2}}{1-q^{n}}
$$


By [13], the generating function of the first positive crank moments for overpartitions can be represented as

$$
\frac{(-q)_{\infty}}{(q)_{\infty}} \sum_{n=1}^{\infty} \frac{(-1)^{n+1} q^{n(n+1) / 2}}{1-q^{n}}
$$

We believe that $h(q)$ should be an important object in the study of $q$-hypergeometric series, automorphic forms and partitions theory.

Now we state our main results, we think they are strange and interesting.

Theorem 1.1. (1) For $X>0$,

$$
\frac{X}{2}-\left(\frac{3}{2}+\sqrt{2}\right) \sqrt{X}-1-\frac{3 \sqrt{2}}{2}<\#\{1 \leq n \leq X \mid h(n)=0\}<\frac{3 X}{4}-\frac{\sqrt{X}}{2 \sqrt{2}}+1 .
$$

(2) For $n=p^{d}$, we have $h(n)=0$, where $p$ is an odd prime and $d$ is an odd positive integer.

(3) For $n=p_{1} p_{2} \ldots p_{k}$, we have $h(n)=0$, where $p_{i}$ are different odd primes, and $p_{k}>2 p_{1} p_{2} \ldots p_{k-1}, k \geq 2$.

Remark. Unlike $\sigma(q)$, which is lacunary, $h(q)$ is not lacunary.

Theorem 1.2. (1) For sufficient large $X$,

$$
\frac{\sqrt{X}}{\log X} \ll \#\{1 \leq n \leq X \mid h(n)=1\}<\left(1+\frac{1}{\sqrt{2}}\right) \sqrt{X}
$$

(2) for $n=2^{k}$, we have $h(n)=1$, where $k \geq 1$ is a positive integer.

(3) For $n=p^{2 k}$, we have $h(n)=1$, where $p$ is an odd prime and is a positive integer.

(4) For $n=p_{1}^{2} p_{2}^{2}$ with $p_{1}$ and $p_{2}$ odd primes and $p_{2}>\sqrt{p_{1}}$, we have $h(n)=1$.

Theorem 1.3. $\quad$ (1) For sufficient large $X$,

$$
\frac{\sqrt{X}}{\log X} \ll \#\{1 \leq n \leq X \mid h(n)=2\}<\frac{X}{2}+\left(\frac{3}{2}+\sqrt{2}\right) \sqrt{X}+1+\frac{3 \sqrt{2}}{2} .
$$

(2) For $n=p_{1} p_{2}$ with $p_{1}$ and $p_{2}$ odd prime and $p_{1}<p_{2}<2 p_{1}$, we have $h(n)=2$.

(3) For $n=p \times(p+1)$ or $(p-1) \times p$, with $p$ an odd prime, we have $h(n)=2$.

Theorem 1.4. For $n=p_{1}^{2} p_{2}^{2}$ with $p_{1}$ and $p_{2}$ odd prime and $p_{1}<p_{2}<\sqrt{2} p_{1}$, we have $h(n)=3$.

Remark. Our theorems above show that $h(n)$ can attain $0,1,2,3$ infinite many times. Numerical experiments suggest more. See the conjectures in Section 3.

Theorem 1.5. $h(n)$ is odd if and only if $n$ is a square or a double square, hence for almost all positive integers $n, h(n) \equiv 0(\bmod 2)$.

Remark. The partition function $p(n)$, its values distributions are simple, just growing fast, but has strange parity (conjecturally equi-distributed see [1], [6], [19]). The distributions of the values of $h(n)$ are strange, but $h(n)$ has simple parity. 
The paper is organized as follows. In Section 2, we prove our main theorems. In Section 3, we give further conjectures about the distributions of the values of $h(n)$.

\section{Proof of the Main theorems}

We start with representing $h(q)$ as

$$
h(q)=\sum_{n=0}^{\infty} h(n) q^{n}=\sum_{n=1}^{\infty} \sum_{j=1}^{n-1} 2 q^{n(n+j)}+\sum_{n=1}^{\infty}\left(q^{n^{2}}+q^{2 n^{2}}\right) .
$$

This is the Lemma 2.2 in [3]. Define three sets $A_{n}, B_{n}$ and $C_{n}$ as follows:

$A_{n}=\{(x, y) \mid n=x(x+y)$, with $x, y$ are positive integers and $1 \leq y \leq x-1$.

$$
\begin{gathered}
B_{n}=\left\{x \mid n=x^{2}, \text { with } x \text { is an positive integer } .\right\} \\
C_{n}=\left\{x \mid n=2 x^{2}, \text { with } x \text { is an positive integer } .\right\}
\end{gathered}
$$

By (2.1),

$$
h(n)=2 \# A_{n}+\# B_{n}+\# C_{n} .
$$

Proof of Theorem 1.1. (1) We note that $h(n)=0$ if and only if $n$ is neither a square nor a double square or does not appear in the following sequeences of numbers for any $m \geq 1$,

$$
m^{2}+m, m^{2}+2 m, m^{2}+3 m, \ldots, m^{2}+(m-1) x .
$$

So $h(n) \neq 0$ if and only if $n$ appears in the following numbers for some $m$,

$1, \quad 2$,

$2^{2}, \quad 2^{2}+2, \quad 2 \times 2^{2}$,

$3^{2}, \quad 3^{2}+3, \quad 3^{2}+2 \times 3,2 \times 3^{2}$,

$\begin{array}{llll} & \cdots & \cdots & \ldots\end{array}$

$m^{2}, \quad m^{2}+m, \quad m^{2}+2 m, \quad m^{2}+3 m, \quad \ldots, \quad 2 m^{2}$.

For sufficient large $X$, let $m$ be the positive integer such that

$$
2 m^{2} \leq X<2(m+1)^{2},
$$

that is

$$
\sqrt{\frac{X}{2}}-1 \leq X \leq \sqrt{\frac{X}{2}} .
$$

Then from 1 to $X$, the number of $n$ such that $h(n) \neq 0$ is at least

$$
\begin{aligned}
& 2+3+4+\cdots+m+(m+1) \\
& =\frac{(m+1)(m+2)}{2}-1 \\
& \geq \frac{X}{4}+\frac{1}{2 \sqrt{2}} \sqrt{X}-1
\end{aligned}
$$


by (2.3). Hence the number of $n$ such that $h(n)$ is zero is at most

$$
\frac{3 X}{4}-\frac{1}{2 \sqrt{2}} \sqrt{X}+1 \text {. }
$$

This prove the inequality of the right hand side of (1.2).

For the inequality of the left hand side of (1.2), let $k$ be the maximal positive integer such that $(m+k)^{2} \leq 2(m+1)^{2}$, note that besides the numbers listed above, some other numbers $n$ still can be $\leq X$ and $h(n) \neq 0$, but they must appear in the following sequences.

$$
\begin{array}{ccccc}
(m+1)^{2}, & (m+1)^{2}+1 \times(m+1), & (m+1)^{2}+2 \times(m+1), & \ldots, & (m+1)^{2}+\times(m+1)^{2}, \\
(m+2)^{2}, & (m+2)^{2}+1 \times(m+2), & (m+2)^{2}+2 \times(m+2), & \ldots, & (m+2)^{2}+\times(m+2)^{2}, \\
\ldots & \ldots & \ldots & \ldots \\
(m+k)^{2}, & (m+k)^{2}+1 \times(m+k), & (m+k)^{2}+2 \times(m+k), & \ldots, & (m+k)^{2}+\times(m+k)^{2} .
\end{array}
$$

Hence, the number of $n$ such that $1 \leq n \leq X$ and $h(n) \neq 0$ is at most

$$
\begin{aligned}
(2+3+4+\cdots+m+(m & +1))+((m+1+1)+(m+2+1)+\cdots+(m+k+1)) \\
& =\frac{(m+k+1)(m+k+2)}{2}-1 \\
& =\frac{1}{2}(m+k)^{2}+\frac{3}{2}(m+k) \\
& \leq \frac{X}{2}+\left(\frac{3}{2}+\sqrt{2}\right) \sqrt{X}+1+\frac{3 \sqrt{2}}{2},
\end{aligned}
$$

by the inequalities $(m+k) \leq \sqrt{2}(m+1)$ and $m+1 \leq \sqrt{\frac{X}{2}}+1$. This is equivalent to the inequality of the left hand side of (1.2).

(2) For $n=p^{d}$ with $p$ an odd prime and $d$ an odd positive integer, it is only to show $A_{2}(n)$ is empty. Suppose the equation $p^{d}=x(x+y)$ has a solution $(x, y)$ with $x>1$ and $x-1 \geq y \geq 1$. We must have

$$
x=p^{d_{1}}, y=p^{d_{2}} \quad \text { with } \quad d_{2}>d_{1} .
$$

This implies that

$$
y=p^{d_{2}}-p^{d_{1}}=p^{d_{1}}\left(p^{d_{2}-d_{1}}-1\right)>2 p^{d_{1}}=2 x,
$$

since

$$
d_{2}-d_{1} \geq 1, \quad p \geq 3 .
$$

This contradicts the assumption $y<x-1$.

(3) for $n=p_{1} p_{2} p_{3} \ldots p_{k}$ with $p_{i}$ distinct odd primes and

$$
p_{1}<p_{2}<p_{3}<\cdots<p_{k-1}, \quad p_{k}>2 p_{1} p_{2} \ldots p_{k-1},
$$

$n$ is neither a square nor a double square, it is only to show $A_{2}(n)$ is empty. Suppose the equation

$$
p_{1} p_{2} p_{3} \ldots p_{k}=x(x+y)
$$


has a solution $(x, y)$ with $x>1$ and $x-1 \geq y \geq 1$. We consider two cases. If $p_{k}$ is a prime divisor of $x$, then we have

$$
x \geq p_{k}>2 p_{1} p_{2} p_{3} \ldots p_{k-1} \geq 2(x+y) .
$$

Hence $y \leq-\frac{x}{2}$, this is impossible. If $p_{k}$ is a prime divisor of $x+y$, then we have

$$
x+y \geq p_{k}>2 p_{1} p_{2} p_{3} \ldots p_{k-1} \geq 2 x,
$$

so $y>x$. This also contradicts the assumption.

These complete the proof of Theorem 1.1.

Proof of Theorem 1.2. (1) By ([2.2), $h(n)=1$ if and only if $A_{n}$ is empty and $n$ is a square or double square. From 1 to $X$, the number of squares is about $\sqrt{X}$ and the number of double squares is about $\sqrt{\frac{X}{2}}$. Hence the number of $1 \leq n \leq X$ such that $h(n)=1$ is at most $\left(1+\frac{1}{\sqrt{2}}\right) \sqrt{X}$. On the other side, for any prime $p$, the sets $B_{p^{2}}, C_{p^{2}}$ are all empty, hence for each $n=p^{2}$ with $1 \leq p^{2} \leq X$, we have $h(n)=1$. By The Prime Number Theorem, the number of squares of primes is about $\frac{\sqrt{X}}{\log \sqrt{X}}=\frac{2 \sqrt{X}}{\log X}$. This completes the proof of the part (1).

(2) If $n=2^{k}, k \geq 1$, we must have $A_{n}$ is empty. Since if we have a solution $(x, y)$ such that

$$
2^{k}=x(x+y), \quad 1 \leq y \leq x-1,
$$

then we can assume that

$$
x=2^{k_{1}}, x+y=2^{k_{2}}, k_{2}>k_{1} .
$$

Therefore,

$$
y=2^{k_{2}}-2^{k_{1}}=2^{k_{1}}\left(2^{k_{2}-k_{1}}-1\right) \geq 2^{k_{1}}=x,
$$

which contradicts the assumption that $1 \leq y \leq x-1$. Moreover, If $k$ is even, then $n$ is a square. If $k$ is odd, then $n$ is a double square. These complete the proof of part (2) of the Theorem[1.2.

(3) For $n=p^{2 k}$ with $p$ odd prime and $k$ positive integer, $n$ can not be a double square and there is no solution $(x, y)$ with $1 \leq y \leq x-1$ such that $p^{2 k}=x(x+y)$. Otherwise, we can assume that

$$
x=p^{k_{1}}, x+y=p^{k_{2}}, k_{2}>k_{1} .
$$

As before

$$
y=p^{k_{2}}-p^{k_{1}}=p^{k_{1}}\left(p^{k_{2}-k_{1}}-1\right) \geq 2 p^{k_{1}}=2 x,
$$

which is not possible.

(4) For $n=p_{1}^{2} p_{2}^{2}$ with the assumption that $p_{1}$ and $p_{2}$ are odd primes with $p_{2}>\sqrt{2} p_{1}$. it is only to show that the equation

$$
2^{k}=x(x+y), \quad 1 \leq y \leq x-1,
$$

has no solutions. We note that $x$ can be $p_{1}$ or $p_{1}^{2}$. for the case $x=p_{1}$, we have

$$
x+y=p_{1} p_{2}^{2} \text {, hence } y=p_{1}\left(p_{2}^{2}-1\right)>2 p_{1} .
$$


For the case $x=p_{1}^{2}$, we have $x+y=p_{2}^{2}$, hence $y=p_{2}^{2}-p_{1}^{2}>p_{1}^{2}=x$, since $p_{2}>\sqrt{2} p_{1}$. This shows the equation above has no solutions. The proof of Theorem 1.2 is completed.

Proof of Theorem 1.3. (1) We note that

$$
\#\{1 \leq n \leq X \mid h(n)=2\}<\#\{1 \leq n \leq X \mid h(n) \neq 0\} .
$$

The right hand side of the inequality above is at most

$$
\frac{X}{2}+\left(\frac{3}{2}+\sqrt{2}\right) \sqrt{X}+1+\frac{3 \sqrt{2}}{2}
$$

which is proved in part one of theorem 1.1. As for the left hand side of (1.3), this follows from the part (3) of the current theorem. Since from 1 to $X$, there are about $\frac{\sqrt{x}}{\log \sqrt{X}}$ numbers which are the forms $p(p+1)$ by the Prime Number Theorem.

(2) For $n=p_{1} p_{2}$ with $p_{1}<p_{2}<2 p_{1}$. Firstly, by a theorem of Betrand-Chebyshev, for any prime $p_{1}$, there is at least one $p_{2}$ satisfying $p_{1}<p_{2}<2 p_{1}$. Moreover, $n$ can not be a square or a double square and the equation

$$
p_{1} p_{2}=x(x+y), \quad 1 \leq y \leq x-1
$$

has a unique solution $(x, y)=\left(p_{1}, p_{2}\right)$ by the condition $p_{1}<p_{2}<2 p_{1}$. Hence $h(n)=2$.

(3) For $n=p(p+1)$ with $p$ odd prime. since $p$ and $p+1$ are coprime, hence $n$ can not be a square or a double square. Consider the equation

$$
p(p+1)=x(x+y), \quad 1 \leq y \leq x-1,
$$

the only solution is $x=p$ and $y=1$, therefore $h(n)=2$. The analysis for the case $n=(p-1) p$ is similar.

Proof of Theorem 1.4. (1) For $n=p_{1}^{2} p_{2}^{2}$ with $p_{1}$ and $p_{2}$ odd primes and $p_{1}<p_{2}<$ $\sqrt{2} p_{1}$, by a theorem in [12], for any sufficient large $p_{1}$, there is at least one prime $p_{2}$ satisfying $p_{1}<p_{2}<\sqrt{2} p_{1}$. $n$ can not be a double square, it is only to show there is only one pair $(x, y)$ with $1 \leq y \leq x-1$ satisfying the equation

$$
p_{1}^{2} p_{2}^{2}=x(x+y) .
$$

Here, $x$ can be $p_{1}, p_{1}^{2}, p_{2} . \quad x=p_{1}$ is impossible, this results in $y=p_{1}\left(p_{2}^{2}-1\right)>$ $2 p_{1}=2 x . x=p_{2}$ is also impossible, it also results in $y=p_{2}\left(p_{1}^{2}-1\right)>2 p_{2}=2 x$. The only solution to the equation above is $(x, y)=\left(p_{1}^{2}, p_{2}^{2}\right)$, because of the condition $p_{1}<p_{2}<\sqrt{2} p_{1}$.

Proof of Theorem 1.5. By (2.1),

$$
h(q) \equiv \sum_{n=1}^{\infty}\left(q^{n^{2}}+q^{2 n^{2}}\right) \quad(\bmod 2),
$$

$h(n)$ is odd if and only if $n$ is a square or a double square and the destiny of square numbers and double square numbers is zero. Therefore, for almost all integers $n, h(n)$ is even. 


\section{QUESTIONS FOR FURTHER STUDY}

We use some quadratic equations to study the small values of $h(n)$, but it is unclear whether there is a relation between the function $h(q)$ and quadratic fields. This is worth investigating. For the distributions of the coefficients of $h(q)$, the experiments suggest more should be true. Here we list our conjectures based on our experiments.

Conjecture 1. $h(n)$ can attain each positive integers infinite many times, In particular, we have $\lim \sup h(n)=\infty$.

This conjectural property for $h(n)$ is similar to that of $S(n)$, which is our original motivating of investigating the function $h(q)$. However, the following conjecture shows $h(q)$ is completely different from $\sigma(q)$.

Conjecture 2. For sufficient large $X$,

$$
\#\{1 \leq n \leq X \mid h(n)=0\} \asymp \frac{3 X}{4}-\frac{1}{2 \sqrt{2}} \sqrt{X},
$$

Conjecture 3. For sufficient large $X$,

$$
\#\{1 \leq n \leq X \mid h(n)=2\} \asymp \frac{X}{4}+\frac{1}{2 \sqrt{2}} \sqrt{X} .
$$

This implies that almost all nonzero values are 2 .

Our Theorem 1.5 coincides with the Conjecture 1 and Conjecture 2.

Conjecture 4. For sufficient large $X$,

$$
\#\{1 \leq n \leq X \mid h(n)=1\} \asymp\left(1+\frac{1}{\sqrt{2}}\right) \sqrt{X} .
$$

More difficult questions are what are the asymptotical behaviors of

$$
\#\{1 \leq n \leq X \mid h(n)=2 k\}
$$

and

$$
\#\{1 \leq n \leq X \mid h(n)=2 k+1\}
$$

for a fixed positive interer $k$ and sufficient large $X$ ?

\section{ACKNOWLEDGEMENTS}

The author would like to thank Professor Peter Paule for his valuable comments on an earlier version of this paper and encouragements. 
TABLE 1 . The number of $1 \leq n \leq X$ such that $h(n)$ takes values from 0 to 16 for $X \leq 100000$.

\begin{tabular}{|c|cccccc|}
\hline$h(n)$ & $X=1000$ & $X=5000$ & $X=10000$ & $X=20000$ & $X=50000$ & $X=100000$ \\
\hline 0 & 664 & 3486 & 7068 & 14312 & 36249 & 73130 \\
1 & 44 & 93 & 129 & 179 & 275 & 380 \\
2 & 255 & 1181 & 2300 & 4455 & 10718 & 20798 \\
3 & 9 & 23 & 32 & 46 & 74 & 108 \\
4 & 27 & 180 & 369 & 758 & 1944 & 3969 \\
5 & 0 & 3 & 7 & 11 & 21 & 31 \\
6 & 1 & 32 & 80 & 186 & 509 & 1051 \\
7 & 0 & 1 & 2 & 5 & 8 & 14 \\
8 & 0 & 1 & 13 & 41 & 148 & 354 \\
9 & 0 & 0 & 0 & 0 & 3 & 5 \\
10 & 0 & 0 & 0 & 7 & 43 & 120 \\
11 & 0 & 0 & 0 & 0 & 0 & 1 \\
12 & 0 & 0 & 0 & 0 & 8 & 28 \\
13 & 0 & 0 & 0 & 0 & 0 & 0 \\
14 & 0 & 0 & 0 & 0 & 0 & 10 \\
15 & 0 & 0 & 0 & 0 & 0 & 0 \\
16 & 0 & 0 & 0 & 0 & 0 & 1 \\
\hline
\end{tabular}

\section{REFERENCES}

[1] S. Ahlgren: Distribution of parity of the partition function in arithmetic progressions, Indag. Math. (N.S.) 10 (1999), 173-181.

[2] G. E. Andrews, Questions and conjectures in partition theory, Am. Math. Monthly 93 (1986), 708-711.

[3] G.E. Andrws, S.H. Chan, B. Kim, R. Osburn, The first positive rank and crank moments for overpartitions, Ann Comb. (2016), DOI 10.1007/s00026-016-0306-0.

[4] G.E. Andrews, S.H. Chan, B. Kim, The odd moments of ranks and cranks, J. Combin. Theory Ser. A 120 (2013), no. 1, 77-91.

[5] G. Andrews, F. Dyson, and D. Hickerson, Partitions and indefinite quadratic forms, Invent. Math. 91 (1988), pages 391-407.

[6] B. Berndt, A.J. Yee and A. Zaharescu: On the parity of partition functions, Internat. J. Math. 14 (2003), no. 4, 437-459.

[7] K. Bringmann and B. Kane, Multiplicative q-hypergeometric series arising from real quadratic fields, Trans. Amer. Math. Soc. 363 (2011), no. 4, 2191-2209.

[8] K. Bringmann and B. Kane, New identities involving sums of the tails related to real quadratic fields, Ramanujan J. 23 (2010), no. 1-3, 243-251.

[9] K. Bringmann, J. Lovejoy and L. Rolen, On some special families of q-hypergeometric Maass forms, arXiv:1603.01783

[10] H. Cohen, q-identities for Maass waveforms, Invent. Math. 91 (1988), pages 409-422.

[11] D. Corson, D. Favero, K. Liesinger, and S. Zubairy, Characters and $q$-series in $\mathbb{Q}(\sqrt{2})$, J. Number Theory 107 (2004), pages 392-405.

[12] H. Kadiri and A. Lumley, Short effective intervals containing primes, Integers 14 (2014), \#A61.

[13] A.Larsen, A. Rust and H. Swisher, Inequalities for positive rank and crank moments of overpartitions, Int. J. of Number Theory, 10 (2014), no. 8, 2115-2133.

[14] J. Lovejoy, R. Osburn, Real quadratic double sums, Indag. Math. 26 (2015), 697-712. 
[15] J. Lovejoy, Lacunary partition functions, Math. Res. Lett. 9 (2002), pages 191-198.

[16] J. Lovejoy, Overpartitions and real quadratic fields, J. Number Theory 106 (2004), pages 178186.

[17] J. Lovejoy, Bailey pairs and indefinite quadratic forms, J. Math. Anal. Appl. 410 (2014), 10021013.

[18] R. Odoni, On the norms of integers in a full module of an algebraic number field and the distribution of values of binary integral quadratic forms, Mathematica 22 (1975), pages 108-111.

[19] K. Ono: Parity of the partition function, Adv. Math. 225 (2010), no. 1, 349-366.

[20] S. Ramanujan, The lost notebook and other unpublished papers, Narosa, New Delhi, 1988.

[21] S. Zwegers, Mock theta functions, Ph.D. Thesis (Advisor: D. Zagier), Universiteit Utrecht, (2002).

[22] S. Zwegers, Mock Maass theta functions, Q. J. Math. 63 (2012), no. 3, 753-770.

Research Institute for Symbolic Computation, Johannes Kepler University, Altenberger Strae 69, A-4040 Linz, Austria

E-mail address: xxiong@risc.uni-linz.ac.at 